Revista Iberoamericana, Vol. LXXIV, Núm. 222, Enero-Marzo 2008, 1-

\title{
DIEZ PREGUNTAS A NOÉ JITRIK
}

\author{
POR \\ Eleni Kefala \\ Universidad de St. Andrews, Escocia
}

Noé Jitrik es profesor, investigador, escritor y director del Instituto de Literatura Hispanoamericana de la Facultad de Filosofía y Letras de la Universidad de Buenos Aires. Es uno de los críticos argentinos de más larga y respetada trayectoria con numerosos ensayos sobre literatura, crítica literaria y teoría. Ha recibido varios premios y honores, entre otros el de Chevalier des Arts et des Lettres (Francia, 1993) y el Premio Xavier Villaurrutia (México, 1981) por sus relatos de Fin del ritual. Actualmente dirige una obra monumental, la Historia crítica de la literatura argentina, publicada por Emecé Editores y de la cual han aparecido seis volúmenes. La magnitud del trabajo, cuyo primer tomo apareció en 1999, se hace evidente en la cifra de los colaboradores (unos 250 ensayistas) tanto como en su distribución internacional (América Latina, Europa y EE.UU.). En esta charla, Jitrik habla de los desafíos de este proyecto gigantesco pero también de sus intereses como crítico, de los cambios a que se ha sometido su punto de vista a lo largo de las últimas tres décadas, de Borges, y de la producción literaria en la Argentina antes y después de la última dictadura. Se pregunta si sirve hoy el concepto civilización y barbarie y razona sobre la noción de lo clásico, las corrientes críticas, el universo lector y editorial, y la crítica endógena y exógena. Además reflexiona sobre autores olvidados y abusados, escritoras mujeres, el diálogo intelectual con Europa y los EE.UU., y qué significa escribir hoy en ese rincón del mundo que se llama Argentina.

Eleni Kefala: Duke University Press acaba de publicar en inglés una colección de ensayos sobre la literatura hispanoamericana intitulada The Noé Jitrik Reader. Ud. ha escrito esos ensayos a lo largo de las últimas tres décadas. ¿Piensa que su punto de vista ideológico e interpretativo ha cambiado a través de los años? Por ejemplo, ¿cómo leía a escritores como Borges en los años setenta, mientras estaba en el exilio en México, y cómo los leería hoy?

NoÉ JiTRIK: No sé si ha cambiado mucho mi punto de vista. Quizás le hayan llegado algunos instrumentos que tal vez, ojalá, lo hayan redondeado, perfilado un poco. Pero habría que definir cuál era ese punto de vista inicial para ver qué es lo que se le puede haber añadido. Y el punto de partida era la posibilidad de intuir en un texto algún elemento significativo que podría explicar lo que es la estructura en general o, con menor ambición, una situación textual determinada. En esa posibilidad de intuición reconozco un poco la 
presencia de Auerbach, el autor de Mimesis -yo no lo hice como lo hizo brillantemente él, pero no dudo que haya en mis trabajos algún resto de su manera. En mi caso, una vez intuido un núcleo, que puede ser un aspecto o un detalle, o una rugosidad textual, traté de elaborarlo hasta llegar a construir o articular un modelo del texto cuyo requisito principal era ético, no traicionado, y fecundo, o sea que tuviera como consecuencia la posibilidad de postular una interpretación.

Pese a los años que transcurrieron, creo que no abandoné esa idea inicial pero debo admitir que se fue felizmente saturando, recibiendo materia teórica o filosófica; comprendí, creo, que para intuir con mayor riqueza, puesto que no existe la intuición salvaje, es preciso enriquecer la mirada. Por otra parte, eso que llamo “intuición” siempre está vinculado con un orden de saber que viene de lecturas, de experiencias, de verificaciones, de simpatías también: es un hecho que a uno le impresionen ciertos referentes y otros no. En ese sentido, reconozco la importancia que tiene el pensamiento de alguien como Michel Foucault, pero debo declarar que nunca me ha contagiado. En cambio, Maurice Blanchot o Roland Barthes sí me han impregnado, o sea, desde mi perspectiva, me han enriquecido. ¿Por qué mi simpatía por una propuesta y no por otra? No lo puedo explicar y eso me aísla porque pareciera que en mi campo académico una posición semejante, tan pretendidamente personal, no puede ni debe sostenerse; en ciertos momentos no apelar a determinado teórico parece subversivo o al menos insólito.

Volviendo al tema, quiero decir que la intuición sostuvo mi camino de lectura, una lectura minuciosa, cada vez más exigente, base de la construcción a la que me referí y cuyo objetivo es determinar cómo el texto va a alguna parte, se inscribe en un universo de significación. Eso prosiguió pero en la última década hubo una variante: tratar de extraer de los textos algunos núcleos pero, al indagar en ellos, determinar su alcance y su validez más allá de los textos en los que residan, como cuestiones de orden filosófico más general. Quizás en The Noé Jitrik Reader este movimiento se advierte en parte; por ejemplo -no es lo que más me interesa por supuesto-en el trabajo titulado "De la historia a la escritura". Yo diría que el arranque de este artículo es una reflexión que sale de una experiencia de lectura de novela histórica pero que tiende a definir ese concepto en su alcance mayor. Posteriormente, y siguiendo esta variante, mis intereses se fueron haciendo más abstractos. No es que desdeñe los textos, pero lo que más me llama la atención en ellos son los conceptos que salen de ellos y no ellos en sí mismos, eso que se llamaba en otra época "crítica inmanente". En un libro que probablemente salga en Francia y cuyo título es Verde es toda teoría, los temas son, por ejemplo, el “referente”, la noción de "significación”, el concepto de "verdad", a los que siempre se vuelve aunque con diferentes miradas; la mía, creo, tiene un tinte semiótico pero no greimasiano, basado en la tendencia a recoger tales temas en ciertos constructos que están en todas partes y en ninguna al mismo tiempo; mi ejemplo predilecto en ese sentido es la “conversación” que, no es difícil afirmarlo, no está en ninguna parte y está en todas. De un interés inicial por la conversación, considerada semióticamente, pasé a otros análogos, el silencio o la exclusión o el secreto que son, a mi ver, núcleos semióticos, como objetos-no objetos, no obstante su carácter, significantes. Estas son las cuestiones que me han ido atrayendo.

En cuanto a la segunda parte de la pregunta, Borges para mí fue un objeto de interés muy temprano, tanto la poesía como los ensayos. Los cuentos que no obstante conocía me 
arredraron por mucho tiempo, sin poder leerlos, sin poder saborearlos. La poesía, en cambio, me conducía, me hacía soñar, allanaba el camino de mi deseo. Además Borges fue como un pequeño río que se juntó con otros dos ríos para darme a mí la posibilidad de pensar en términos de poesía: me refiero a T. S. Eliot y Guillaume Apollinaire, tan diferentes. Los poemas de los tres se me mezclaron y me dijeron, los tres al mismo tiempo, que escribiera poesía. Escribí poesía, no importa, les debo eso, o sea que Borges ocupa un lugar importante en mi trabajo. Eso no quiere decir que no haya pensado en los textos borgeanos sino que simplemente quiero decir cuál es mi inclinación actual, o sea qué escribo cuando escribo.

Después me interesaron los ensayos. Cuando apareció Otras inquisiciones yo escribí un articulito muy tempranamente, era un iniciado. Después Borges entró en un cono de sombra por dos razones. La primera porque estábamos todos en otra línea de pensamiento, yo también, más politizante. El concepto de literatura era difícil de asumir. Siempre es difícil decir qué es literatura pero hay una percepción de lo que es y a veces hay un período muy largo en el que uno rechaza esa percepción, prefiere quedarse con un sistema puramente referencial y no problematizarse. Luego, en mi período protoestructuralista -nunca fui estructuralista del todo-Borges empezó a inquietarme cuando me metí con sus narraciones; empecé a verlo con otros ojos y traté de organizar los cuentos de Ficciones y, mediante ese gesto, de comprenderlos. Después de la incisión que sufre mi espectro teórico a causa del psicoanálisis, se me produce otra variante, aunque quiero aclarar que no se trató nunca de hacer psicoanálisis literario, o sea determinar qué pasaba con el autor de un texto. Lo que de entrada me tentó fue establecer una homología entre psicoanálisis y literatura en la medida en que ambas actividades, o sea tipos de discurso, implican una hermenéutica y comparten la materia, o sea el lenguaje. Ese cruce reorganizó mi percepción de los textos y me sirvió para escribir un trabajo sobre García Márquez, otro sobre Roberto Arlt y, por fin, en términos más teóricos, sobre la relación misma entre psicoanálisis y literatura.

Desde ese mirador, incitado por una publicación relacionada con la cultura argentina en la época de la dictadura, me refiero a un número especial de Les Temps Modernes, escribí un largo artículo en el que intentaba saldar cuentas con Borges: me refiero a mi ensayo "Sentimientos complejos sobre Borges". Posteriormente hubo nuevos escritos sobre Borges, más ocasionales, más requeridos, algún congreso, alguna reunión, cosas por el estilo. Tengo no obstante la impresión de que el proceso de configuración de mi mirada crítica reaparece en esas intervenciones de una u otra forma, o bien se manifiesta en ellas. Desde luego que no sé si he descubierto algo nuevo en los últimos trabajos que hice. Me impresiona la riqueza de Borges pero también me pregunto cuándo esa riqueza se va a agotar porque no hay nada humano que no se agote. Lo que queda en todo eso es que su obra por ahora es una caja fuerte llena de cosas que se pueden ir retirando, no sé hasta cuándo. Eso ahí guardado es siempre interesante, importante y valioso: Borges tuvo intuiciones literarias de primer orden que no tienen una formulación canónica y por eso persisten, porque supo cómo darles una forma insólita. Yo puedo ver esto desde un afuera pero también se fue configurando adentro de mí de manera inconsciente o en el inconsciente, según lo prevé el psicoanálisis. Pero debo decir, por otra parte, que gran 
parte del trabajo borgeano de organización, de perspectiva, de desarrollo o de modo de ver, tiene sus orígenes en la relación, tanto de Borges - muy notoria-como mía con Macedonio Fernández: eso en común es lo que quizás me permite leerlo. En cuanto a Borges, de quien se podría decir que poseía un inconsciente muy vigoroso, si se permite la broma, vio en el discurso literario infinidad de aspectos, con absoluta precisión, fuerza y rigor. En fin, creo que eso es lo que funda este hecho completamente extraordinario de la difusión que ha tenido.

EK: ¿Cree que la caída de la última dictadura en la Argentina ha generado algún cambio significativo en las corrientes literarias del país en las últimas dos décadas?

NJ: Yo creo que sí porque si las líneas de fuerza de una literatura son, por un lado una tradición fuerte que para algunos empieza con Esteban Echeverría y sigue con Eugenio Cambaceres, con Manuel Gálvez, con Roberto Arlt, hasta llegar a Julio Cortázar y Ernesto Sábato, por el otro hay otra línea, justamente la que encarnan Macedonio Fernández y Borges: yo creo que ambas líneas se andan persiguiendo. Hay intersecciones, desde luego, escritores que posteriormente al momento fuerte de presencia de esas corrientes las vinculan y producen algo nuevo: me refiero a Alberto Vanasco o a Antonio di Benedetto, por ejemplo; en apariencia parecen incluirse en la línea más tradicional pero al mismo tiempo es evidente en sus textos una impronta vanguardista muy fuerte. Y, puesto que se menciona a la dictadura, me parece que esto que acabo de señalar conforma el debate literario argentino previo a la dictadura. Durante la dictadura la producción literaria olvida esos dilemas, se sofoca un poco o permanece a la sombra o se va al exterior; es el caso de Osvaldo Lamborghini, un vanguardista radical. Pero el dilema no desaparece: hay un evidente juego entre esas tradiciones en una novela como Respiración artificial, de Ricardo Piglia: hasta cierto punto se afilia a la línea borgeana pero, por otro lado, reivindica la tradición de tipo realista, arltiana. Hay que señalar, no obstante, que la feliz fórmula de Piglia recupera también pero tenuemente una tradición realista que, por su irresistible tendencia al denuncialismo y la atención a lo social-político, no se animaba en tales condiciones de censura y represión a salir a la luz del día.

Entre tanto, puede decirse que hay un auge de la literatura de importación; el bestseller traducido se impone con mucha fuerza, aunque el fenómeno sea poco importante en el sentido de la literatura o de una historia de la literatura. De hecho, es un fenómeno social, sociológico y mercadológico, más que literario. Pero, complementariamente, el bestseller tiene otras consecuencias: impone modelos literarios norteamericanos, estilísticos y éticos, de cierta ética de la escritura. Yo creo que después de la dictadura esa presencia parece ofrecer una salida para muchos de nuestros escritores que quieren volver al realismo, que será realismo más bien a la norteamericana: sintaxis directa y de impacto, temática convenientemente elegida para hacerse entender sin problemas por un público que sufre frente a la dificultad de leer, acercamiento a la experiencia vivida, frases cortas y eficaces, escasas descripciones, mucha acción. Los escritores más conocidos están en esa línea. Por fin, creo que el culto al lenguaje de la literatura norteamericana supone por cierto el abandono del modelo francés, o sea esa poética que hacía de la literatura un objeto escultórico y uno de cuyos últimos representantes podría decirse que era Adolfo Bioy Casares. 
Idelber Avelar, un brillante crítico brasileño, acuñó una frase que me parece feliz: la literatura posterior a la dictadura es una literatura en duelo. O sea que asume, como duelo, todo lo que caducó, todo lo que murió durante la dictadura pero también, como luego de toda muerte, en parte niega eso mismo. ¿En qué consiste el duelo? Para proponer ese concepto Idelber Avelar parte de los textos de Tununa Mercado, donde se tematiza la pérdida, pero eso sería lo más evidente. Lo menos evidente sería otra pérdida, la pérdida de confianza en la lengua y en la palabra en otro nivel; la desconfianza se impone en todos los órdenes, un rasgo posmoderno, si quiere, pero que no se alimenta de la filosofía de la posmodernidad sino que sale de la experiencia contemporánea. Para mí la literatura en la que vibra el duelo en todas sus dimensiones es la mejor, la más consistente, la que siento que conserva todavía esa pasión por una palabra que se evade pero que, por eso mismo, es trabajada, significada, una palabra concentrada y no simplemente la importación de episodios relacionados con temas interesantes de la realidad inmediata, destinada a cautivar lectores primarios.

EK: ¿'Piensa que la tensión entre civilización y barbarie sigue teniendo un papel significativo en la Argentina contemporánea o bien, ahora que el edificio logocéntrico ha sido socavado por los “indecidibles" derridianos, estos términos ya han sido transgredidos?

NJ: La sarmientina pareja civilización y barbarie no sería más que un esquema servicial e indicativo del momento histórico en el que se formuló, no una verdad esencial, si las hay. Tuvo éxito, muchos la reprodujeron para sus propias necesidades de análisis pero posteriormente se la vivió como muy abusiva, como muy injusta pero, no obstante, sea como fuere, forma parte del pensamiento de América Latina, no sólo de la Argentina. Incluso se la pudo haber vivido como un sustituto del concepto de la lucha de clases. La Revolución Mexicana, por ejemplo, fue vista por la clase dominante como barbarie enfrentada con la civilización porfiriana; la escasa viabilidad del concepto, en ese mismo caso, es puesta rápidamente en evidencia, no sólo por lo que la Revolución dejó sino por el papel que desempeñó un intelectual como José Vasconcelos quien, de formación "civilizada", adhiere a esa presunta "barbarie" y genera un plan cultural de extraordinario humanismo. En suma, ¿quién define lo que es la civilización y lo que es la barbarie, qué es el terrorismo, fácilmente calificable como expresión de barbarie? Puras contradicciones: los principales actores del nazismo, que en su momento pretendían representar la civilización (porque sostenían que los judíos, gitanos, homosexuales, etcétera, eran infrahumanos), tuvieron en los hechos un comportamiento esencialmente inhumano, equivalente a lo contrario de la civilización. Conclusión: esta terminología no sirve o sirve poco o confunde.

Lo que en cambio me parece que corresponde es tratar de entender ciertas emergencias en el orden político. Por ejemplo, la elección de Evo Morales en Bolivia. Desde el punto de vista de los Patiño que dominaron Bolivia en la década del treinta y con el producto del saqueo construyeron castillos y coleccionaron obra pictórica, Evo es un mero indio, bárbaro, sin cultura ninguna. ¿Pero por qué de pronto este hombre llega -no digo que tenga grandes éxitos ni que los pueda tener- a desempeñar un papel histórico? Es sin duda un hecho nuevo como lo es la emergencia en América Latina de personajes, aunque no sean comparables, que llegaron a la presidencia y que poco tiempo antes eran desconocidos u oscuros, como Menem, Chávez, el ecuatoriano que cantaba rock, el mexicano gerente de 
la Coca-Cola, el olvidable Collor de Mello y así siguiendo. ¿Cómo entender esas emergencias? Por un lado está la idea de un cansancio discursivo y, complementariamente, la sofocante sensación de inmovilidad histórica. Si esto es así, se debe haber producido en cada caso una ansiedad por algo nuevo y diferente aunque de allí no se saque que se crea demasiado en aquello a que puede dar lugar: si bien la credibilidad es una cosa y la ansiedad es otra se diría que son primas hermanas. La ansiedad, lo saben los psiquiatras, es mala consejera, puede llevar a decisiones fatales para el que, sobre esa sensación, las toma pensando que al hacerlo disminuye la angustia que la suele acompañar. A esto hay que añadir que en el horizonte de decisiones tomadas así, se produce una suspensión del razonamiento, en el que se apoya otro impulso, el de la posibilidad de que alguien, cualquiera, nos saque de esta situación: ahí llega la credibilidad y con ella un casi seguro regreso a lo mismo. Me parece entonces que el lugar común "civilización y barbarie” no es válido, no deja pensar.

Mencioné algo al pasar y me gustaría no abandonarlo, me refiero a la noción del terrorismo. Creo que el terrorismo tiene dos fuentes, no una sola. Una es, como señalé, la angustia de vastos sectores de la población, incluso políticos. Por ejemplo, ¿cuál era la angustia en la época en que surgía la clase obrera y que dio lugar al anarquismo? Este fenómeno, ligado a la naciente revolución industrial, fue en su momento considerado terrorismo; es cuestión, entonces, de una necesidad que llega a formularse y que descansa en bases reales de experiencia.

La otra fuente es la vocación y el imaginario. La vocación hace que el imaginario se ponga a buscar elementos para darle satisfacción. Pero el imaginario no necesariamente es convertible ni universal y los medios para lograr esa satisfacción no son siempre los mejores. En ese cruce, la noción del terrorismo empieza a ser un interpretante y eso es lo que desconcierta y desbarata los análisis porque no se puede decir que poner una bomba en el metro de Londres sea defendible, ni válido el argumento de que eso es una respuesta a la invasión de Irak. La pregunta subsiste: ¿por qué ponen bombas? Me supongo que es porque imaginan que es un medio adecuado para canalizar la relación entre vocación e imaginario. Y cuando digo vocación digo también "misión”. ¿Ignoro este concepto? Metido en este cuarto, mirando libros que no leí todavía y que no sé si voy a leer, con una máquina que me está llamando y que recibe de mi parte pobres respuestas, no me atrevería a decir que hay que poner bombas o que hay que hacer esto o lo otro para arreglar el mundo; no entra en mi cabeza tal grandiosa misión. Diría, en consecuencia, que el tema reside en un imaginario en el que descansan los elementos que se supone que permiten ejecutar la misión. Lamentablemente es un imaginario que empiezo a creer que es prodigioso porque el que se haya descubierto que mezclando dos líquidos se puede destruir un avión es impresionante, semejante al descubrimiento de la dinamita. En definitiva, lo que desconcierta es esa misionalidad. Así como desconcierta en el orden religioso. ¿Por qué fulano quiere ser cura? ¿Qué es lo que lo lleva a ser cura? Ser abogado o escritor puede ser una vocación pero que sea una misión es difícil considerarlo mientras que poner bombas e inmolarse con ellas parece ser una misión.

EK: ¿Quiénes serán los clásicos de mañana? ¿Destacan hoy en día, en su opinión, algunos escritores en Argentina o en otros países de Hispanoamérica que podrían alcanzar o a lo mejor superar la fama de los clásicos latinoamericanos? 
NJ: Eso es muy delicado y difícil de responder así sea porque ha cambiado sustancialmente tanto el universo lector como el paraliterario, o sea, editorial, y tiende a imponerse la noción del consumo. Es probable por lo tanto que algunos libros, revolucionarios en el sentido en que hubo libros revolucionarios en el siglo diecinueve o al principio del veinte, pasen inadvertidos, que sean deglutidos y desaparecidos rápidamente. Ahí hay, para la determinación de la existencia de algo que puede ser clásico, una noción de paciencia histórica y esto es muy difícil de pedir porque un libro que sale en la Argentina demora en las librerías dos o tres semanas, y si no se vende de entrada desaparece. ¿Quién lo puede recuperar si se publican por año en la Argentina centenares de libros? Es muy difícil y en todas partes es lo mismo. En virtud de ese criterio de realidad se producen ciertas operaciones que podemos llamar "ortopédicas", por ejemplo que una editorial movilice todos sus recursos para sostener un libro, ya sea porque supone que se va a vender, ya sea porque estima a un autor (que va a vender). Mediante ese mecanismo puede ser que impongan un libro pero de ahí a que se prediga que va a ser un clásico, hay un trecho, sólo la historia lo puede franquear.

La pregunta sería ¿quién decide que un libro sea un clásico, el lector o el crítico? En verdad no se sabe quién es “el lector” ni dónde está, ni cómo va a manifestarse como tal. Es el texto lo que lo convoca y suscita y si esa atracción da un fruto, nace el lector y al mismo tiempo nace una lectura, tanto más verdadera cuanto más diferente, capaz de reconocer la densidad, la importancia, la trascendencia de unos de esos objetos perdidos en el mar de las comunicaciones. Desde luego que todo escritor aspira a ser clásico, pero ¿quién lo va a conseguir? Yo acabo de publicar un libro que se llama Atardeceres. Cuando se presentó en Rosario, un poeta extraordinario que se llama Mario Trejo, lo blandió y exclamó: ¡esto es un clásico! Su argumento fue compartido por tres personas que lo compraron, entre los cuarenta asistentes. ¿Clásico? No sé. Si Mario Trejo hubiera sido Gastón Gallimard y hubiera dicho es un clásico y lo vamos a publicar en la Pleiade y además en relación con una editorial japonesa, tal vez en lugar de tres lo habrían comprado seis o alguno más, seducido por tan brillante proposición. No creo que se pueda decir mucho más sobre este tema, sólo reflexiones como estas, un poco tristes y que ponen en evidencia la distancia que hay entre nuestro deseo de inmortalidad como escritores y las posibilidades de satisfacerlo.

EK: ¿Considera que hay diferencias importantes entre la crítica literaria endógena y exógena sobre la literatura argentina?

NJ: Sí, yo creo que hay diferencias. La crítica exógena tiende a ser muy académica, cuantitativa, estadística, descriptiva, valorativa y creo que la endógena tiende a ser más cuestionadora, más reflexiva y más sensible a la incorporación de aparatos analíticos, en lo posible cada vez más sofisticados. Yo lo advierto en la gente que trabaja en el Instituto (de Literatura Hispanoamericana) conmigo. Son unos cuantos, es decir, es un banco de prueba bastante bueno: empezaron su carrera, en su mayoría, haciendo trabajos de corte y alcances universitarios sobre autores, períodos, temas y textos, y poco a poco fueron refinándose, produciendo discurso crítico de altura. ¿En qué consiste el refinamiento crítico? No hay una única respuesta: algunos dirigen una mirada más poética, otros una mirada más semiótica, otros una mirada más psicoanalística, etcétera. En fin, hay una pluralidad de posibilidades y elecciones pero lo común en todas ellas es que tienden a 
salirse de la calle universitaria aun estando en la Universidad mientras que numerables tesis de doctorado escritas en los EE.UU. sobre escritores argentinos son casi meras descripciones. Es difícil encontrar algo que pueda ser recuperado, productivo y original.

EK: Actualmente Ud. está realizando un gran proyecto que para muchos sería un desafío imposible: una historia panorámica y al mismo tiempo minuciosa de la literatura argentina que consiste en varios volúmenes. ¿Cuáles son los riesgos que corre un proyecto como ese y cuáles son sus objetivos?

NJ: El primer objetivo es el más elemental de las historias que es dar a conocer algo. El segundo objetivo es darlo a conocer con ciertos criterios, con cierta filosofía. Por lo tanto no se trata de pura recuperación sino de parcial recuperación. Luego se trata también de dar cuenta de cuáles son los puntos de vista que se fueron instalando en el universo literario argentino. Así que son varias cosas al mismo tiempo. Cada una de ellas tiene su explicación pero todo confluye en la idea de una historiografía posible cuyo núcleo ideológico descansa en una genérica definición: toda historia es un relato; en este campo, se relatan textos -no lo que los textos relatan- y por lo tanto, como todo relato, éstos son finalmente producto de determinados y complejos procesos. Así, entonces, cada volumen se articula en torno a uno de esos procesos e intenta cercarlo. Uno y otro dan cuenta -es lo que intentamos- de lo que pasó, y eso es lo que intenta toda historia, en esa entidad que se llama literatura argentina. Obviamente, se trata de determinar explícitamente cuáles son los aspectos de ese proceso y de hacer pensar sobre cada uno. Desde luego, el lenguaje que resulta tiene, para usar un término convencional, un pronunciado carácter ensayístico que, sin embargo, evita las constricciones genéricas porque restringe en lo posible el campo de las opiniones y de las valoraciones maniqueas, la tiranía del gusto como fuente de valoración, las fobias. De lo que se trata es de lograr que haya una concentración discursiva, incluso una identidad discursiva en tan vasto espectro. Yo creo que lo estamos logrando por lo menos en un sesenta o setenta por ciento, no me atrevería a decir un cien. El proyecto es ambicioso pero a mí me parece muy natural. También pienso que esta empresa sea quizás o bien prematura o bien, al mismo tiempo, anacrónica porque muchos no entienden la propuesta y otros creen que no hay por qué ejecutarla; de ahí que, con tristeza, verifico que no se está discutiendo su alcance aunque este objeto producido debería dar lugar a una discusión, así sea porque las relaciones que proponemos nunca fueron consideradas por las historias de la literatura, por ejemplo el periodismo, ciertas comunidades lingüísticas, incluso discursos en apariencia muy alejados, como el jurídico, todo lo cual puede ser visto como un abuso, como un forzamiento, pero no es así porque si el discurso jurídico es el discurso de la ley, el discurso literario también la implica.

EK: ¿Cuáles son los escritores que la crítica ha dejado injustamente al margen del canon literario y cuáles son aquellos de los que los críticos y los académicos han “abusado” en el siglo $\mathrm{xx}$ ?

NJ: De Borges han abusado, de Cortázar han abusado, ahora en ese momento están abusando de Saer y de Piglia. Habrán abusado de muchos autores en su momento, supongo que han abusado de Roberto Arlt, también nosotros cuando estamos preparando un volumen sobre Macedonio Fernández nos estamos abusando de él, pero simultáneamente hay escritores que han sido olvidados. Siempre me surge como ejemplo de autor olvidado 
Arturo Cerretani, un escritor muy importante, o el poeta Mario Trejo, y así siguiendo, la lista de autores olvidados es muy grande. Claro que olvidados por la crítica o la historia, el olvido del universo lector no cuenta en este razonamiento. También están los que son abusados a medias por ciertos círculos que naturalmente tienen sus dioses. En cuanto a la crítica, en la universidad aparecen siempre algunos teóricos canonizados, abusados, pero, dicho sea en homenaje a la moda que obliga a invocarlos o soportarlos, son episódicos: en una época no tan lejana Walter Benjamin era indispensable, lo mismo que Umberto Eco o que Roland Barthes o, más recientemente, Gilles Deleuze o Jacques Derrida. Ocurre algo semejante en la crítica feminista, Julia Kristeva, así como Luce Irigaray, aparece siempre, es como si hubieran formado el canon teórico. Por supuesto habrá otros productores de teoría que en algún momento se convertirán en abusados según lo determinen las corrientes académicas.

EK: El canon literario argentino está lleno de textos escritos por hombres y la literatura argentina, con pocas excepciones, ha alcanzado una fama internacional gracias a aquellos. ¿Quiénes son las escritoras que verdaderamente han tenido o tienen una influencia notable en el terreno literario del país?

NJ: Yo en vez de "influencia” diría "presencia”. Algunas son muy conocidas, como Silvina Ocampo, y otras no. Una escritora de primer orden que no tiene tanta presencia es Libertad Demitrópulos, con una obra buenísima. Muchas están sostenidas por otras escritoras lo que no indica necesariamente que tengan presencia en un universo más amplio. Para muchos, Alejandra Pizarnik tiene una presencia muy fuerte pero para mí su figura está idealizada, es una diosa que trasciende el género, me refiero al femenino. Hay en el volumen 11 de nuestra Historia crítica de la literatura argentina un artículo exhaustivo sobre mujeres escrito por María Rosa Lojo. Es un catálogo de todas las que escriben. Ahí se puede ver, quizás calificar, quién tiene más o menos presencia. En un ciclo de conferencias que se hicieron en la Biblioteca Nacional, Tununa Mercado habló sobre las "raras", o sea escritoras que desafían todas las convenciones. Su presencia, en el sentido de circulación o atención periodística y aun crítica, es escasa, pero en la mirada de alguien que quiere ver un poco más lejos su presencia es innegable.

EK: Argentina, quizá más que ningún otro país latinoamericano, siempre estuvo en contacto con movimientos estéticos foráneos y sobre todo europeos. ¿Cuál es la situación hoy? ¿Sigue existiendo este diálogo trasatlántico?

NJ: Menos creo, pero también más calificado porque depende del campo de lectura. Así, por ejemplo, hay ciertos poetas que conocen a Odiseo Elytis. ¿Qué quiere decir eso? Elytis no viene de Francia, es decir, de una literatura que tradicionalmente tuvo mucha influencia en este país. De la literatura alemana, de pronto aparece alguien como Paul Celan y con él, pero no sólo con él, el interés por el tema de la identidad suspendida por la realidad concentracionaria, un tema que siguieron muchos escritores franceses, italianos, alemanes y otros. La literatura portuguesa empieza a interesar pero no en el sentido en que se pensaba que tradicionalmente operaba, es decir, no como modelos a seguir sino como un diálogo más universal. Con respecto a los EE.UU., hay un modelo que tiene que ver con una sintaxis narrativa y es tan exitoso que muchos tienden a seguirlo. Hablo, por cierto, de los escritores, críticos y universitarios. 
Se podría decir por lo tanto que el diálogo continúa aunque no tenga la evidencia que tuvo cuando la revista Sur y la diligente Victoria Ocampo no sólo traían escritores europeos de prestigio sino que los celebraban brillantemente. De todos modos, la noción misma de diálogo debería ser matizada o entendida al menos en dos planos, visto, además, que mucha gente habla de la existencia de una grave crisis de lectura. Uno de esos planos es el del lector común, que consume, cuando busca lectura, best-sellers: no sabe que está dialogando transatlánticamente; el otro es el del mundo "simbólico", escritores, críticos y universitarios; los primeros se dejan llevar, como siempre, por la buena literatura que se produce en el mundo; los otros suelen responder o replicar manifestaciones críticas exitosas (la importancia que se le da a noción de "canon" o el empleo de la palabra "posmoderno" son buenos ejemplos de la transatlanticidad) o teóricas muy consumibles (Benjamin, Foucault, Deleuze eran y son invocados con tanto respeto que parecen imprescindibles, como parecía imprescindible Homi Bhabha).

Sea como fuere, también es cierto que el diálogo con el pasado continúa: ¿Quién no leyó a Thomas Mann? ¿Quién no leyó a Kafka? ¿Quién no leyó a Joyce o a Proust? En síntesis, yo diría que subsisten líneas secretas que operan en varias direcciones: por un lado, genéricamente, van conduciendo hacia la literatura norteamericana, esencialmente en relación con la sintaxis narrativa y la importancia conferida a lo temático y a la historia, y por el otro lado, hacia la europea, el acento puesto en la búsqueda de la palabra, del lenguaje, en la pasión por la escritura.

EK: Aparte de crítico y académico usted también es escritor. ¿Cómo es escribir en la época de la globalización desde "un suburbio del mundo" como dice Piglia?

NJ: Ni tan suburbio, ni tan mundo (se ríe). La idea de globalización ocupa excesivamente la cabeza de mucha gente. Es verdad -es un lugar común-que hoy es más fácil acceder a cualquier tipo de información: la consecuencia es esa idea de globalización que, por lo menos, me paraliza, no me deja pensar en lo concreto y particular, puesto que en literatura la información es hasta cierto punto irrelevante: lo relevante es la proyección, la trascendencia, lo que los antiguos llamaban la "virtud". Por otra parte, que un escritor, aquí o en Turquía, conduzca su imaginario hacia lo que pide el exceso de información o, lo que es lo mismo, a parámetros admitidos por lo que se llama el "público", sumergido precisamente en el exceso, es garantía segura de que no ingresará en el mundo de la literatura pero también es garantía probable que haga la felicidad de las empresas editoriales. Literatura, u obra, es un objeto que va tomando forma, que no termina de tomar forma, una búsqueda eterna de lo que la palabra oculta o no quiere proporcionar y esto es lo mismo para la Europa de Hegel, centro de la historia, como para la aldea latinoamericana o africana. Para mí escribir en este rincón del mundo está definido por la frase final de “Tlön, Uqbar y Orbis Tertius”, que el narrador escribe después de sopesar un objeto que viene de esos mundos imaginados: "Yo no hago caso, yo sigo revisando en los quietos días del hotel de Adrogué una indecisa traducción quevediana (que no pienso dar a la imprenta) del Urn Burial de Browne". 